\title{
Bayesian Optimization of Computer-Proposed Multistep Synthetic Routes on an Automated Robotic Flow Platform
}

\author{
Anirudh M. K. Nambiar, ${ }^{[[a]}$ Christopher P. Breen, ${ }^{[[b]}$ Travis Hart,, ${ }^{[a]}$ Timothy Kulesza, ${ }^{[a]}$ \\ Timothy F. Jamison, ${ }^{*[b]}$ and Klavs F. Jensen ${ }^{*[a]}$ \\ $\S$ Contributed equally \\ [a] Department of Chemical Engineering, Massachusetts Institute of Technology, \\ 77 Massachusetts Avenue, Cambridge, MA 02139 (USA) \\ [b] Department of Chemistry, Massachusetts Institute of Technology, \\ 77 Massachusetts Avenue, Cambridge, MA 02139 (USA) \\ ${ }^{*}$ Correspondence: kfjensen@mit.edu (K. F. Jensen), thj@mit.edu (T. F. Jamison)
}

\begin{abstract}
Computer-aided synthesis planning (CASP) tools can propose retrosynthetic pathways and forward reaction conditions for the synthesis of organic compounds, but the limited availability of context-specific data currently necessitates experimental development to fully specify process details. We plan and optimize a CASP-proposed and human-refined multistep synthesis route towards an exemplary small molecule, sonidegib, on a modular, robotic flow synthesis platform with integrated process analytical technology (PAT) for data-rich experimentation. Human insights address catalyst deactivation and improve yield by strategic choices of order of addition. Multi-objective Bayesian optimization identifies optimal values for categorical and continuous process variables in the multistep route involving 3 reactions (including heterogeneous hydrogenation) and 1 separation. The platform's modularity, robotic reconfigurability, and flexibility for convergent synthesis are shown to be essential for allowing variation of downstream residence time in multistep flow processes and controlling the order of addition to minimize undesired reactivity. Overall, the work demonstrates how automation, machine learning, and robotics enhance manual experimentation through assistance with idea generation, experimental design, execution, and optimization.
\end{abstract}




\section{Introduction}

Machine assistance has helped automate and accelerate steps in the synthesis of organic compounds, accelerating the discovery and development of new medicines and materials. Going from a molecular structure to a tangible product or fully-defined synthesis route involves two high-level tasks: (1) synthesis planning, where the starting materials, reactions, and reagents that can be used to make the target molecule are identified, and (2) process development, where the unit operations and reaction conditions needed to maximize process performance are specified. Computer-aided synthesis planning (CASP) tools, ${ }^{1,2}$ based on human-curated reaction rules or algorithmically-learned transformations from published reaction data, can automatically generate recommendations for retrosynthetic routes and reaction conditions, helping to generate ideas quickly and reducing manual database lookups. On the process development side, walk-up multistep synthesis platforms ${ }^{3-11}$ equipped with reactors, separators, process analytical technology (PAT), and automation tools (e.g., liquid handlers, reaction sampling, code scripts, user interfaces) enable data-rich experimentation and lighten the manual workload associated with repetitive reaction execution and analysis.

There is growing interest in experimentally validating CASP suggestions and integrating CASP tools with automated synthesis platforms. Experimental validation of CASP recommendations has been demonstrated via batch synthesis ${ }^{12-14}$ and on a robotic continuous flow synthesis platform ${ }^{6}$ and disclosed examples still remain rare. The main obstacle to direct implementation of CASP-designed routes without human intervention is that synthesis procedures require a fully-defined set of instructions (a recipe) specifying the sequence of unit operations and corresponding reaction conditions (e.g., concentrations, temperature, time, stoichiometry) with a level of precision that exceeds what can currently be learned or extracted from limited data. ${ }^{15}$ Therefore, human input was still needed in the reported cases to fill in key procedural details and manually optimize approximate reaction conditions generated by CASP.

Algorithm-guided automated optimization of reaction conditions to maximize a desired objective function (e.g., yield) is another example of machine assistance in organic synthesis. ${ }^{4,16-23}$ In this approach, an algorithm proposes reaction conditions to evaluate within a defined search space (set of values that continuous or categorical process variables can take) based on feedback and analysis of results from previous experiments. Coupling the algorithm with an automated synthesis platform and inline/online PAT tools results in closed-loop design and execution of experiments for efficient reaction development. ${ }^{24-26}$ Prior applications of algorithmic optimization to chemistry have focused primarily on single-step transformations ${ }^{17}$ involving model reactions, with recent work on two-step reaction-separation processes. ${ }^{27,28}$ Furthermore, only a few reports consider categorical reaction variables ${ }^{18,21,22}$ (e.g., catalyst) and multiple objectives. ${ }^{19,22}$ In reality, however, process development for functional organic compounds often involves multi-reaction pathways with categorical reagent choices and several process metrics of interest (e.g., yield, productivity).

In this work, we leveraged algorithm-guided multistep reaction optimization as a tool to identify optimal process conditions for approximate synthesis recipes generated by CASP (Figure 1). The open source CASP software ASKCOS ${ }^{6,29}$ was used to generate recommendations for synthesis routes and reaction conditions for an exemplary small molecule target, sonidegib. After manual assessment of synthetic feasibility, we selected a high-ranked route to optimize involving three reactions and one phase separation. While ASKCOS recommendations and manual solubility testing populated some reaction conditions (solvent, base, catalyst, concentration) in the approximate recipe, specification of critical process parameters was left to a multi-objective Bayesian algorithm that optimized both continuous and categorical variables with respect to multiple process metrics. Experiments proposed by the algorithm were executed on a modular, robotically reconfigurable, continuous flow synthesis platform equipped with multiple PAT tools for reaction analysis and feedback. We consider the chemical and physical interdependencies that arise when optimizing multiple telescoped steps simultaneously and show how the synthesis platform's modularity unlocks an independent degree of freedom for varying downstream reaction time. 


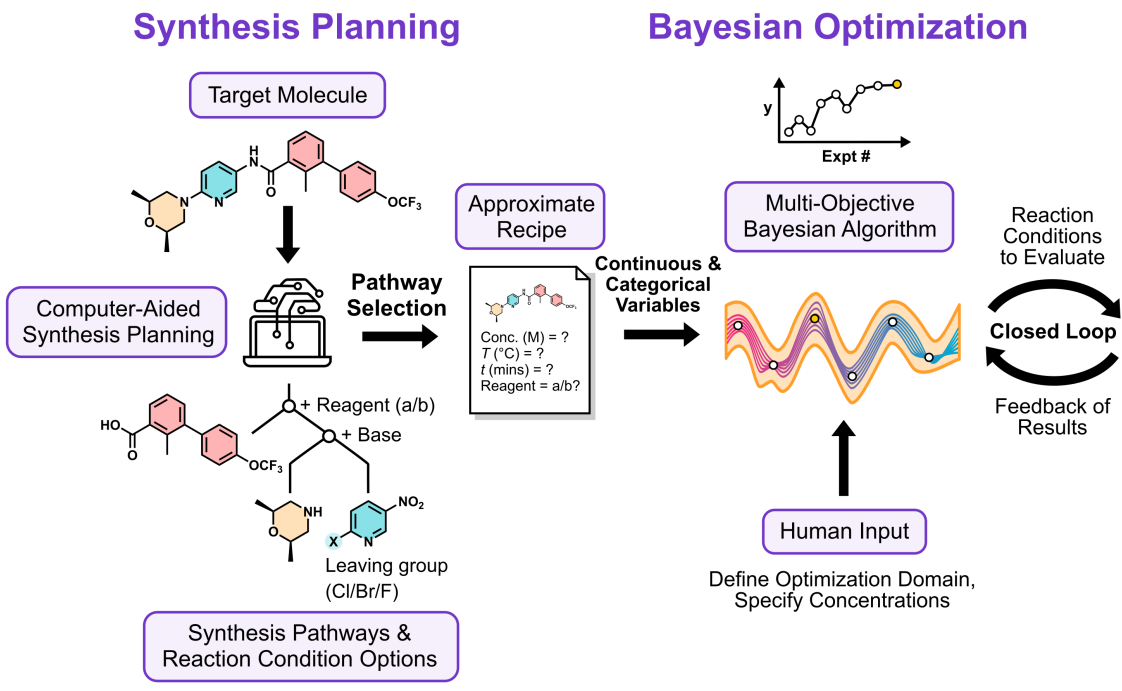

\section{Process Development}

Automated Robotic Platform for Multistep Flow Synthesis \& Reaction Analysis
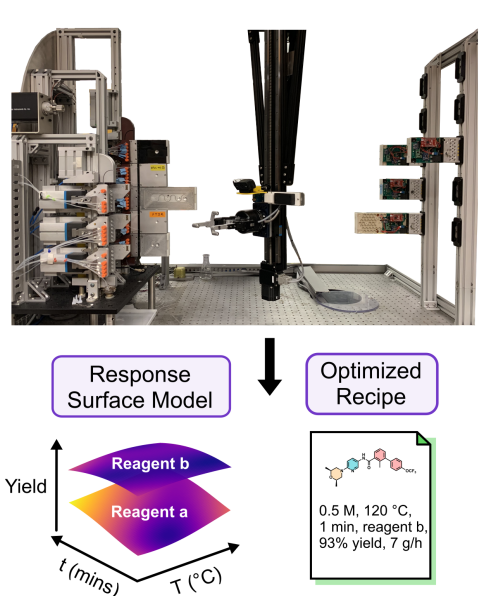

Figure 1. Overall approach for machine-assisted synthesis planning and process development. Computeraided synthesis planning (CASP) recommendations for synthesis routes and reaction conditions to make the target molecule are assessed by humans to create an approximate recipe with missing process details. A multi-objective Bayesian reaction optimization algorithm coupled to a robotic multistep flow synthesis platform optimizes continuous and categorical reaction conditions to fully specify the synthesis recipe.

\section{Results and Discussion}

\section{Computer-Aided Synthesis Planning for Sonidegib}

\section{A) Retrosynthetic Pathways}

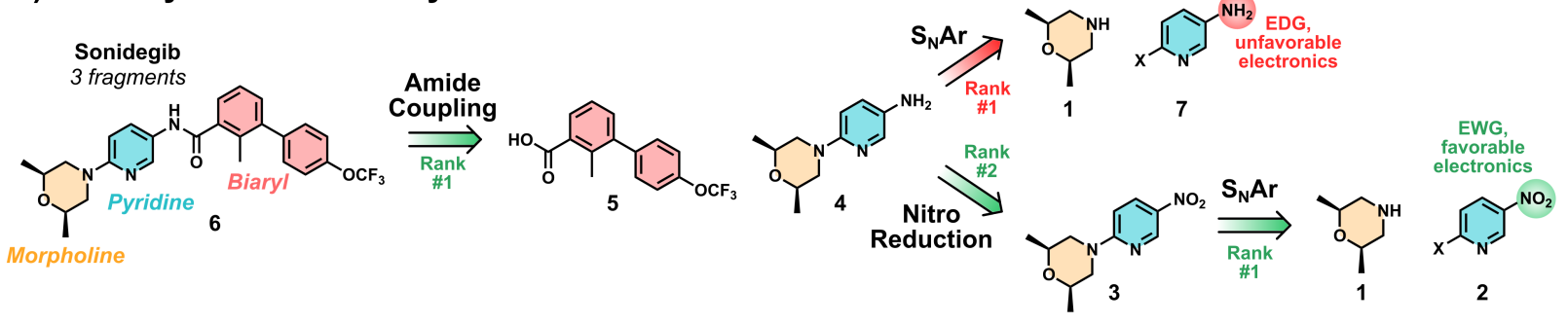

B) Forward Reaction Conditions (Continuous, Categorical)

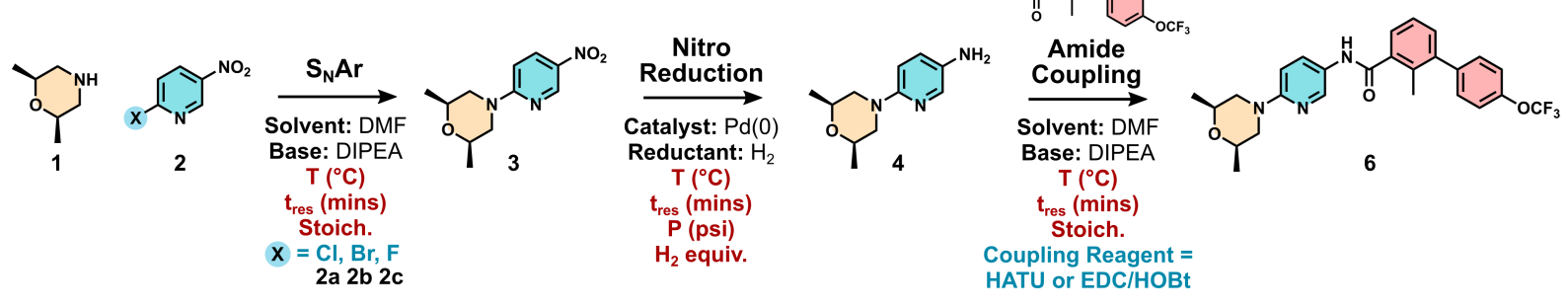

Scheme 1. Computer-aided synthesis planning recommendations for sonidegib 6. (A) Top-ranked retrosynthetic pathways. (B) Proposed forward reaction conditions with continuous variables in red and categorical variables in blue. Abbreviations: EDG (electron donating group), EWG (electron withdrawing group), HATU (hexafluorophosphate azabenzotriazole tetramethyl uronium), EDC (1-ethyl-3-(3dimethylaminopropyl)carbodiimide), HOBt (1-hydroxybenzotriazole). 
The active pharmaceutical ingredient sonidegib 6 (Odomzo $®)^{30,31}$ was chosen as an exemplary target molecule for multistep synthesis. The open source CASP software ASKCOS was used to generate retrosynthetic pathways and forward reaction conditions (Scheme 1, full details in SI). In the rank \#1 pathway, the first retrosynthetic disconnection of 6 occurs at the central amide bond, resulting in substituted aminopyridine 4 and biaryl 5 (Scheme 1A). Aminopyridine 4 may be constructed either in one step via the rank \#1 $S_{N} A r$ reaction of morpholine 1 with a haloaminopyridine 7, or in two steps via the rank \#2 pathway involving reduction of $\mathbf{3}$ and $S_{N} A r$ starting with halonitropyridine 2. The software further proposed that chlorinated $\mathbf{2 a}$, brominated $\mathbf{2 b}$, and fluorinated $\mathbf{2 c}$ were viable starting materials. Structure-reactivity principles suggested that the SNAr reaction of 1 and 7 would be disfavored while the same reaction of 1 and $\mathbf{2}$ would proceed smoothly. Therefore, we decided to proceed with the 3-step route starting from 1 and 2.

In the forward reaction conditions for the 3-step route (Scheme 1B, shown under the arrows), dimethylformamide (DMF) was recommended as the rank \#1 solvent for both the $S_{N} A r$ and amide coupling reactions. For the $S_{N} A r$ reaction, both inorganic and organic amine bases were recommended but solubility testing revealed that DIPEA ( $N, N$-diisopropylethylamine, or Hünig's base) was necessary to fully solubilize the ammonium halide salt byproduct. The need to verify that all components are soluble at the reaction concentration before flow synthesis is a step where human insight is still needed in the current approach. A heterogeneous palladium $(0)\left(\mathrm{Pd}^{0}\right)$ catalyst with $\mathrm{H}_{2}$ as the terminal reductant was proposed for the reduction of 3 . While these details partially populated the synthesis recipe, there were both continuous and categorical reaction conditions (red and blue, respectively, in Scheme 1B) that were left to experimental optimization. These included continuous variables such as temperature, residence time, and stoichiometry, but also categorical choices recommended by ASKCOS such as the $S_{N} A r$ halide leaving group $(\mathrm{Cl}, \mathrm{Br}$, or $\mathrm{F})$ and the amide coupling reagent (HATU or EDC/HOBt).

\section{Robotic Flow Synthesis Platform}
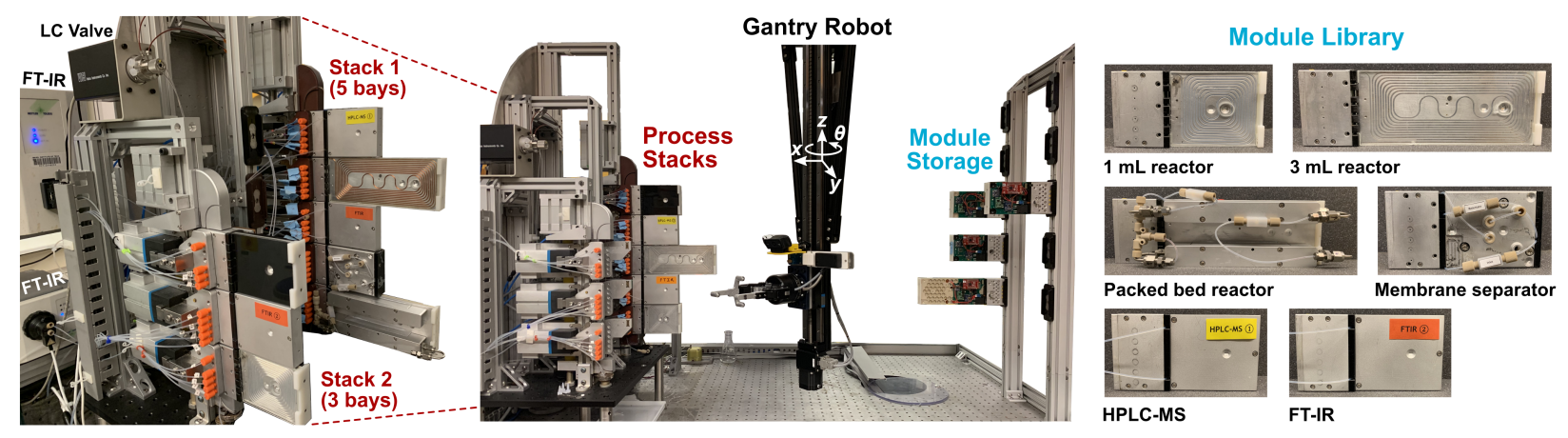

Figure 2. Multistep flow synthesis platform with a library of robotically reconfigurable process modules for reactions, separations, and inline/online analysis.

The CASP-proposed and human-refined synthesis route was experimentally optimized on a modular, robotically reconfigurable, continuous flow synthesis platform (Figure 2) originally developed by our lab ${ }^{6}$ and improved with a faster robot and capabilities for reaction analysis and feedback optimization (see Supplementary Movie and SI for more details). The enabling features of continuous synthesis include access to elevated temperatures and pressures to accelerate reaction rates, tight control over reaction conditions due to shorter length scales for heat and mass transfer, ability to model scaling and mixing behavior due to well-defined reactor geometries, and availability of hardware for inline separations and reaction monitoring. ${ }^{26,32-34}$

Continuous telescoped multistep synthesis, i.e., all steps performed simultaneously in an uninterrupted sequence, ${ }^{35}$ can be realized on the platform by fluidically connecting multiple unit operations together. The platform consists of 2 process stacks with 8 process bays in total, and process modules can be placed by a 4-axis gantry robot ( 3 linear XYZ Cartesian axes +1 rotary $\theta$ axis) onto the process bays in any order to access both linear and convergent sequences. The linear and rotary axes of the gantry robot facilitate 
straightforward path planning (compared to our previous six axis robot) and have a high repeatability (XYZ axes: $5 \mu \mathrm{m}, \theta$ axis: 5 arc-sec) for consistent pick-and-place within 1 minute per process module. Process modules available include heated tubular reactors with various volumes $(0.5,1$, and $3 \mathrm{~mL}$ of PFA tubing), a packed bed reactor, and a membrane phase separator for inline liquid-liquid or gas-liquid separations. The robot also interfaces with a reagent tubing "switchboard" (akin to a telephone wire switchboard) to attach inlets and outlets only to the process bays where they are required. Two of the inlet lines are connected to selector valves that allow switching between different reagent candidates during an experiment.

Three new robotically reconfigurable analytical modules for inline FT-IR spectroscopy and online LC-MS were integrated into the platform enabling data-rich experimentation. The analytical modules contain tubing that directs the process stream either to an external FT-IR instrument with a flow cell or an HPLC sample injection valve connected to an LC-MS, and brings it back to the process stack post-analysis enabling further reactions to be performed if necessary. The modular approach allowed analysis at multiple points in a multistep sequence to quickly obtain critical process performance information such as conversion, yield, and impurity profiles.

\section{Interdependencies in Multistep Flow Processes}

Telescoped flow sequences, analogous to one-pot synthesis in batch, are attractive since they minimize the number of intermediate purification steps required and allow unstable high-potency intermediates to react shortly after formation. However, chemical compatibility of the reagents, solvents, and byproducts must be ensured across all individual unit operations. Optimization of multiple interconnected reactions simultaneously, in comparison to separately or sequentially, reduces the number of optimization campaigns needed, decreases the time and manual effort spent to isolate intermediates, and increases the likelihood of identifying a global system optimum. The process systems engineering literature shows that when there are interdependencies between individual subsystems that affect their respective performance, combining the subsystem optima does not necessarily lead to the global system optimum. ${ }^{36,37}$ In the context of chemical process optimization, examples of interdependencies include cases where excess reagent improves the yield of an upstream reaction but adversely affects the yield of a downstream reaction, or how a certain solvent may be optimal for one reaction but another provides the best overall process yield and ensures solubility. It is therefore beneficial, when possible, to optimize all variables in the overall process simultaneously to identify any interactions and avoid re-optimizing steps.

Another type of interdependency that arises in multistep flow processes is physical constraints on downstream residence times. In continuous reactors, input flow rate and reactor volume determine residence time. While the residence time in the first reactor can be varied independently by changing the flow rate, downstream flow rates are fully specified by stoichiometric relationships with upstream reagents. Consequently, downstream residence times cannot be varied independently. Chatterjee et al. overcame this constraint in their radial synthesis platform ${ }^{8}$ by storing intermediate solutions in an interim vessel until needed for the next reaction. While this approach decouples residence times, it is restricted to chemistries that do not produce unstable or hazardous intermediates that may pose additional challenges upon accumulation and long-term storage. In this work, we leveraged the modular nature and robotic reconfigurability of our synthesis platform to introduce an independent degree of freedom enabling variation of downstream residence time. Specifically, the robot was used to automatically reconfigure downstream reactor volume between a $1 \mathrm{~mL}$ and $3 \mathrm{~mL}$ module on the fly during a multistep optimization campaign. In this way, both shorter and longer downstream residence times could be accessed without altering upstream flow rates.

\section{Bayesian Optimization Algorithm}

The open source Bayesian optimization package, Dragonfly, developed by Kandasamy et al. ${ }^{38-40}$ was chosen as the optimization algorithm (further details in SI). Dragonfly employs a flexible Gaussian process (GP) surrogate model to mathematically describe the relationship between input variables and objective functions. It supports continuous (e.g., temperature), discrete categorical (e.g., leaving group), and discrete numeric (e.g., reactor volume) variables, all of which were present in our case. Another useful feature in 
Dragonfly is the capability to optimize multiple objectives simultaneously, which we availed of to consider multiple process metrics of interest (yield, productivity, cost).

An optimization campaign begins with an initial space-filling design of experiments. After fitting the model to initialization data, the algorithm was queried for one new reaction condition to evaluate at each refinement iteration. Refinement experiments are generated using the upper confidence bound (UCB) and Thompson sampling (TS) acquisition functions which balance an exploitative strategy (query regions where objective is expected to be high) with an explorative one (query regions where uncertainty is high) to increase the likelihood of finding globally optimal points. ${ }^{38,41}$ For multi-objective optimization, the algorithm samples different weights (relative importance) for each objective function at each refinement iteration and maximizes the weighted sum. ${ }^{39}$ If the system contains a Pareto trade-off front ${ }^{16}$ where one objective cannot be improved without making another worse, sampling different weights enables the algorithm to identify multiple Pareto optimal points.

Hardware setpoint control and execution of experimental designs were automated using Python scripts. Once the user specified the process configuration and optimization variable bounds in a spreadsheet file, it was loaded into a graphical user interface (GUI) where backend code parsed this information, queried the algorithm for experimental conditions, calculated flow rates, and generated a queue of commands to set up, execute, and transition between experiments. The GUI automatically read and converted LC peak areas to reaction yields using a pre-determined calibration curve, which closed the information feedback loop and allowed the platform to operate independently once initiated.

\section{Fully Telescoped Process Experiments}

The fully telescoped sonidegib 6 synthesis process was executed on the platform (Figure 3A-B). The first step was performed in a $1 \mathrm{~mL}$ reactor followed by an LC-MS module to determine yield of $\mathrm{S}_{\mathrm{N}} \mathrm{Ar}$ product 3. The platform switched between the three different halonitropyridine starting materials $\mathbf{2 a}, \mathbf{2} \mathbf{b}$, and $\mathbf{2 c}$ via a selector valve. Subsequent reduction to amine 4 was accomplished using the packed bed reactor module wherein $\mathrm{H}_{2}$ was introduced via a mass flow controller and the resulting biphasic gas-liquid stream passed through heated tubes containing a heterogeneous $\mathrm{Pd}^{0}$ catalyst. Hydrogenation reactions generally benefit from continuous processing due to enhanced gas-liquid-solid mass transfer and smaller reaction volumes that improve throughput and safety. ${ }^{42}$ Immediately after the packed bed, an inline membrane separator module removed residual hydrogen gas which exited in the retentate stream since the downstream amide coupling step and reaction sampling modules required a homogeneous liquid stream. The liquid permeate was conveyed to the inline FT-IR module for real-time monitoring of nitro $\mathbf{3}$ and amine $\mathbf{4}$ concentrations. We observed that when exposed to ambient air, solutions of amine 4 changed from colorless to dark red due to amine oxidation on the timescale of minutes. The telescoped flow approach was therefore beneficial in this case for minimizing exposure to oxygen, obviating the need for purification, and ensuring that the unstable amine was utilized promptly after formation. After FT-IR analysis, the reaction stream was delivered to the second process stack where the final amide coupling of $\mathbf{4}$ and $\mathbf{5}$ was conducted in either a $1 \mathrm{~mL}$ or $3 \mathrm{~mL}$ reactor to access different residence times. The overall yield of sonidegib 6 for the three step sequence was determined using the second LC-MS module. Deploying three analytical modules simultaneously enabled data-rich process development, since each experiment provided information about three different reactions. 

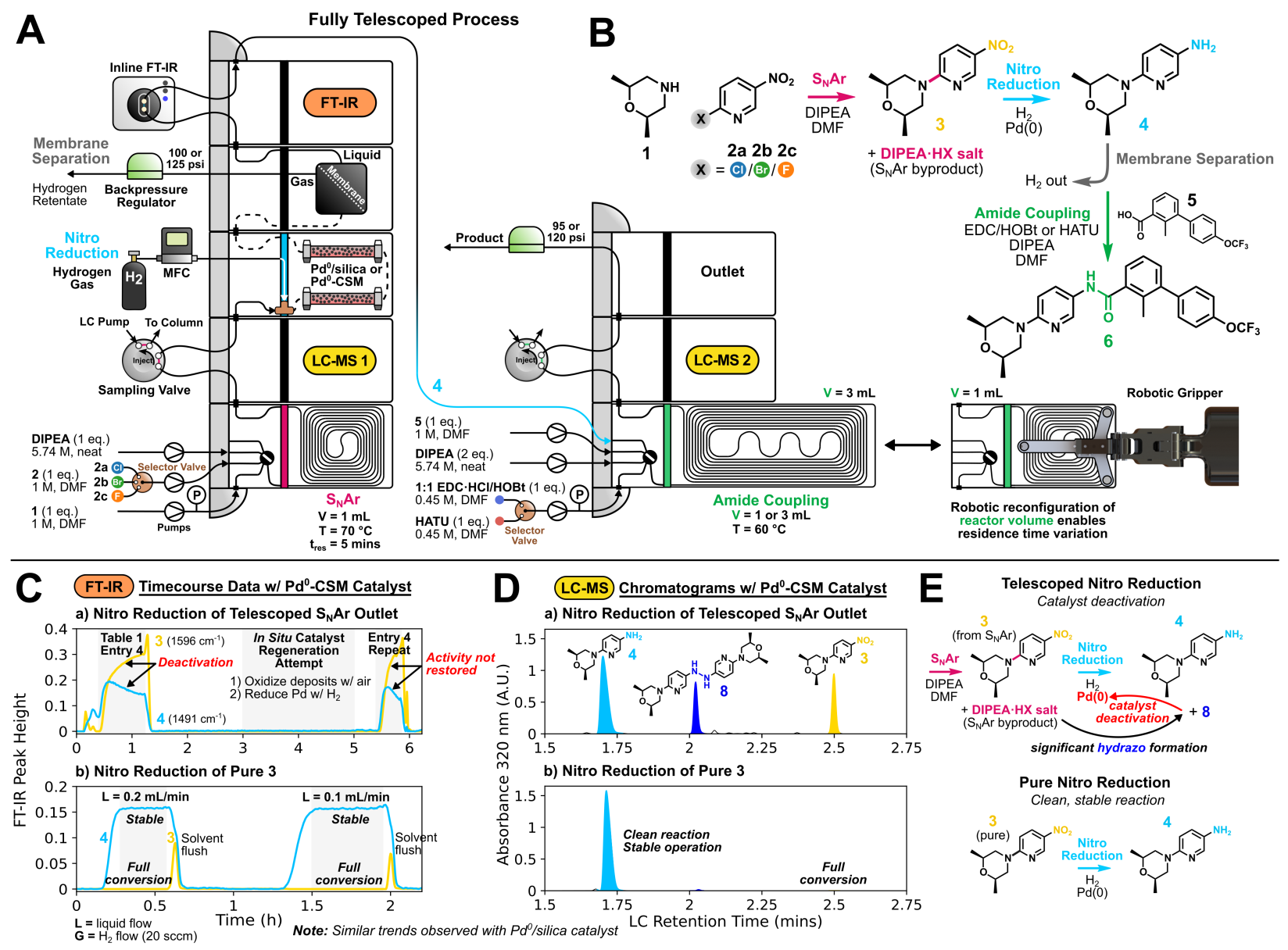

Figure 3. Fully telescoped process experiments for multistep synthesis of sonidegib. (A) Platform configuration. (B) Process scheme. (C) FT-IR timecourse data, (D) LC chromatograms, and (E) schemes of (a) telescoped and (b) pure nitro reduction. Abbreviations: CSM (catalytic static mixer).

Before running a multistep optimization campaign, preliminary experiments (Table 1) were performed to verify that the fully telescoped process and $\mathrm{Pd}^{0}$ catalyst activity were stable. For these preliminary experiments, continuous variable values were typically set near the midpoint of the intended optimization range. Two different catalysts, $\mathrm{Pd}^{0} / \mathrm{silica}^{43,44}$ and $\mathrm{Pd}^{0}$-electroplated stainless steel catalytic static mixers $(\mathrm{Pd}-\mathrm{CSM}),{ }^{45,46}$ were initially investigated.

Table 1. Preliminary experiments with fully telescoped process. ${ }^{[a]}$

\begin{tabular}{|c|c|c|c|c|c|c|c|}
\hline Entry & $\begin{array}{c}S_{N} A r \\
\text { Leaving } \\
\text { Group }\end{array}$ & $\begin{array}{c}\mathrm{S}_{N} \mathrm{Ar} \\
\text { Yield }[\%]^{[b]}\end{array}$ & $\begin{array}{l}\text { Reduction } \\
\text { Catalyst }\end{array}$ & $\begin{array}{l}\text { Coupling } \\
\text { Reagent }\end{array}$ & $\begin{array}{l}\text { Coupling Time } \\
\text { [mins] }\left(\mathrm{V}_{\mathrm{R}}\right)\end{array}$ & 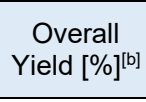 & Observations \\
\hline 1 & $\mathrm{Cl}$ & 84 & $\mathrm{Pd} /$ silica $^{[c]}$ & HATU & $2(1 \mathrm{~mL})$ & 14 & $\begin{array}{l}\text { Incomplete reduction and catalyst } \\
\text { deactivation (even at } 100^{\circ} \mathrm{C} \text { ) }\end{array}$ \\
\hline 2 & $\mathrm{~F}$ & 95 & $\mathrm{Pd} /$ silica $^{[\mathrm{c}]}$ & $\mathrm{EDC} / \mathrm{HOBt}$ & $2(1 \mathrm{~mL})$ & - & $\begin{array}{l}\mathrm{S}_{\mathrm{N}} \mathrm{Ar} \text { fluoride salt byproduct resulted } \\
\text { in disintegration of silica support }\end{array}$ \\
\hline 3 & $\mathrm{~F}$ & 96 & $\mathrm{Pd}-\mathrm{CSM}^{[\mathrm{d}]}$ & $\mathrm{EDC} / \mathrm{HOBt}$ & $2(1 \mathrm{~mL})$ & 17 & SS316L support resistant to fluoride \\
\hline 4 & $\mathrm{~F}$ & 96 & $P d-C_{S M}^{[d]}$ & HATU & $6(3 \mathrm{~mL})$ & 53 & $\begin{array}{l}\text { Catalyst deactivation eventually } \\
\text { observed after few hours }\end{array}$ \\
\hline
\end{tabular}

[a] Fixed process conditions (see Figure $3 \mathrm{~A}): \mathrm{S}_{N} \operatorname{Ar}\left(T=70^{\circ} \mathrm{C}, t_{\text {res }}=5\right.$ mins, 1 equiv 1,1 equiv DIPEA), amide coupling $\left(T=60{ }^{\circ} \mathrm{C}, 2\right.$ equiv DIPEA, 1 equiv coupling reagent). [b] Yield determined by LC with an internal standard. [c] Pd/silica reduction conditions: $0.5 \mathrm{~g}$ catalyst, $T=70^{\circ} \mathrm{C}$, backpressures $=100$ psi retentate, 95 psi outlet, $\mathrm{H}_{2}$ flow $=20 \mathrm{sccm}$ (10 equiv). [d] Pd-CSM reduction conditions: 
two CSMs in series, $T=120^{\circ} \mathrm{C}$, backpressures $=125$ psi retentate, 120 psi outlet, $\mathrm{H}_{2}$ flow $=30$ sccm (15 equiv). Abbreviations: CSM (catalytic static mixer), $V_{R}$ (reactor volume).

The first LC-MS module showed that the $S_{N} A r$ reaction proceeded to $>80 \%$ yield with both leaving groups (Table 1, entries 1-4). However, the inline FT-IR after the reduction step revealed that Pd catalyst deactivation within the timescale of an experiment (tens of minutes to an hour) was a major issue preventing stable operation with either catalyst support. With $\mathrm{Pd} /$ silica, the FT-IR timecourse showed a gradual rise in unconverted nitro starting material $\mathbf{3}$ with a concomitant decrease in amine product $\mathbf{4}$ (similar to Figure $3 \mathrm{C}(\mathrm{a})$ ). While the $\mathrm{Cl}$ leaving group in $\mathbf{2 a}$ resulted only in deactivation, the $\mathrm{F}$ leaving group in $\mathbf{2 c}$ led to disintegration of the silica support (Table 1, entries 1-2). A stoichiometric byproduct of the upstream $S_{N} A r$ reaction is the conjugate acid salt of DIPEA (Figure $3 \mathrm{~B}$ ), and the disintegration of the silica support can be attributed to chemical incompatibility with fluoride.

To solve the chemical compatibility issue, we turned our attention to Pd-CSMs which are 3D printed from chemically resistant stainless steel 316L. ${ }^{45}$ The nitro reduction was operated at the platform's upper limit of $120{ }^{\circ} \mathrm{C}$ to suppress catalyst deactivation. 47,48 When we experimentally evaluated two Pd-CSMs in series with 2c starting material (Table 1, entries 3-4), the stainless steel scaffold was indeed chemically resistant to the DIPEA.HF byproduct but deactivation was eventually observed after a few hours of operation. An attempt to regenerate the $\mathrm{Pd}$ catalyst in situ ${ }^{44,49}$ did not restore the original catalyst activity (Figure $3 \mathrm{C}(\mathrm{a})$ ).

To investigate the cause of deactivation (see SI for further details), only the nitro reduction step was carried out with isolated nitro compound 3. FT-IR and LC-MS data (Figure $3 C(b), D(b)$ ) showed complete conversion and stable performance over multiple hours with both catalysts. While the pure nitro reduction proceeded cleanly, the telescoped reduction outlet contained a significant amount of hydrazo dimer $\mathbf{8}$, an intermediate in the reduction pathway (Figure 3D). ${ }^{50}$ Therefore, the quick onset of catalyst deactivation observed with the telescoped process (Figure $3 \mathrm{E}$ ) was likely due to catalyst poisoning by the $\mathrm{S}_{N} \mathrm{Ar}$ byproduct salt or by strong adsorption of condensation intermediates on the Pd surface. ${ }^{49}$

The ability to integrate analytical modules both within and at the end of the multistep sequence was crucial for identifying which reaction was affecting the overall yield and stability of the fully telescoped process. Due to the catalyst stability issues, it was necessary divide the process into a single-step SNAr optimization with offline purification of $S_{N} A r$ product 3 , followed by a multistep optimization involving reduction of isolated 3, gas-liquid membrane separation, and amide coupling to form sonidegib 6.

\section{$S_{N}$ Ar Multi-Objective Optimization Campaign}

For the $S_{N} A r$ campaign (Figure 4), five optimization variables and three objective functions were optimized simultaneously. The reaction was carried out in a heated $1 \mathrm{~mL}$ reactor module (operated up to the hardware limit of $120^{\circ} \mathrm{C}$ ) followed by an LC-MS module for yield quantification and an FT-IR module for steady-state monitoring (see Supplementary Movie). The optimization domain consisted of four continuous variables (residence time, temperature, equiv of 1 , equiv of DIPEA) and one categorical variable (2a, $\mathbf{2 b}$, or $\mathbf{2 c})$. We considered objective functions related to both reaction performance and raw material costs. Therefore, in addition to reaction yield and productivity (grams product/hour), we included cost as a third optimization objective which was defined as the cost of reagents used per mole of product made. The reagent cost accounted for the different costs of the three starting material candidates (cost $\mathbf{2} \mathbf{a}<\mathbf{2} \mathbf{b}<\mathbf{2} \mathbf{c}$ ) as well as the additional cost of using excess morpholine 1 and DIPEA. 


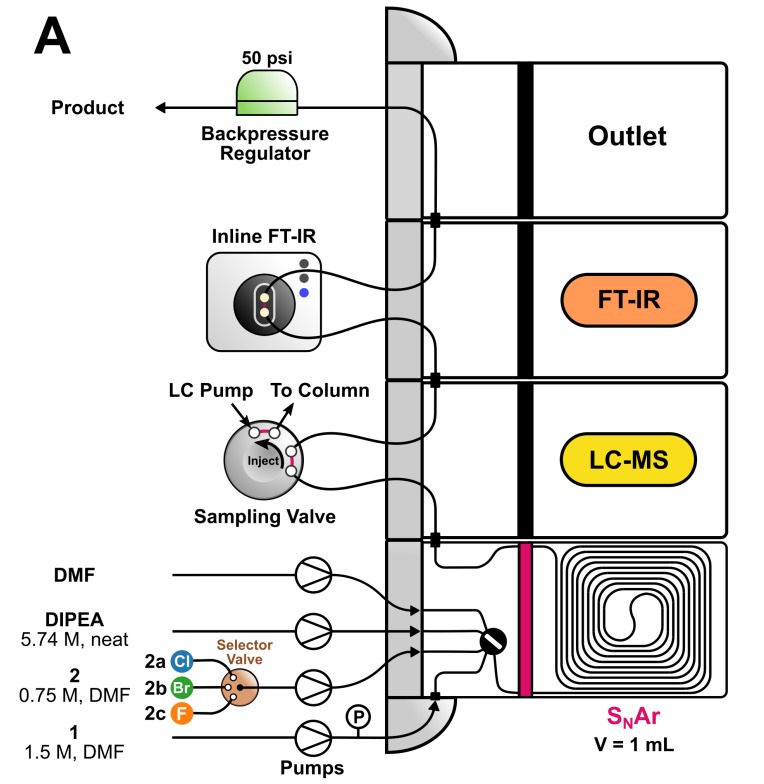

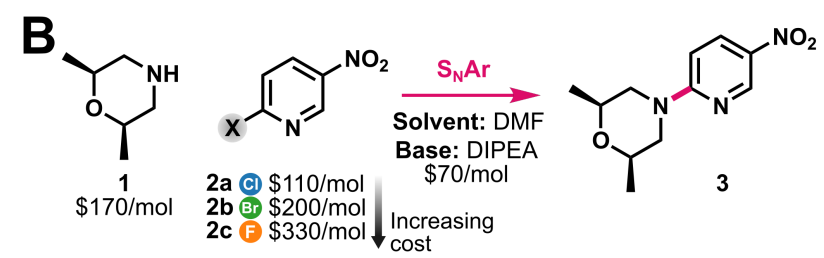
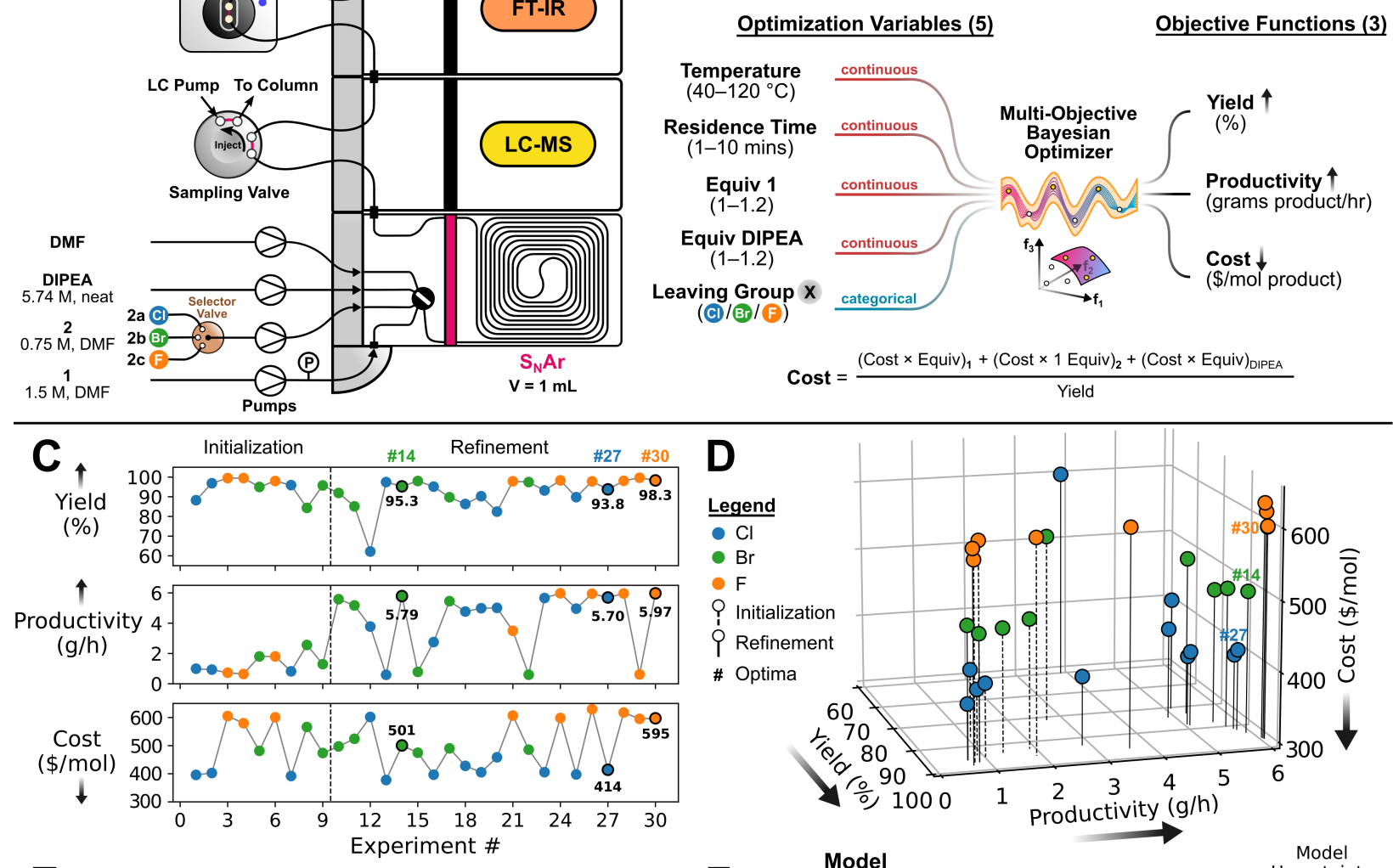

E

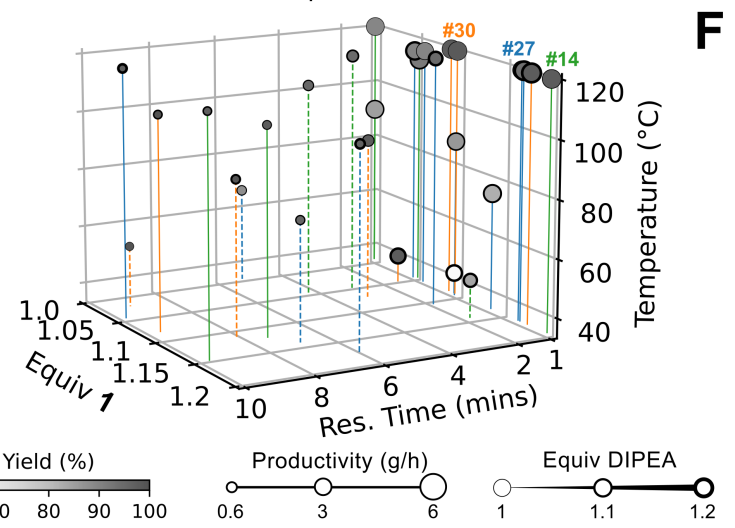

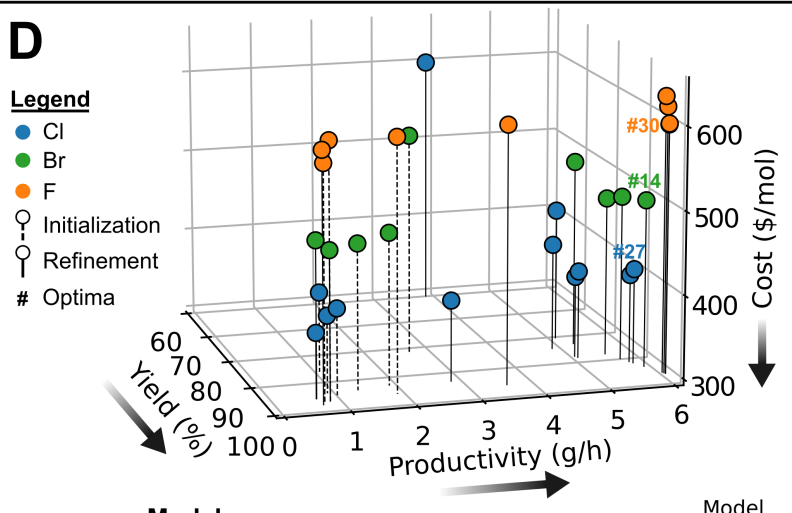

E Response Surfaces

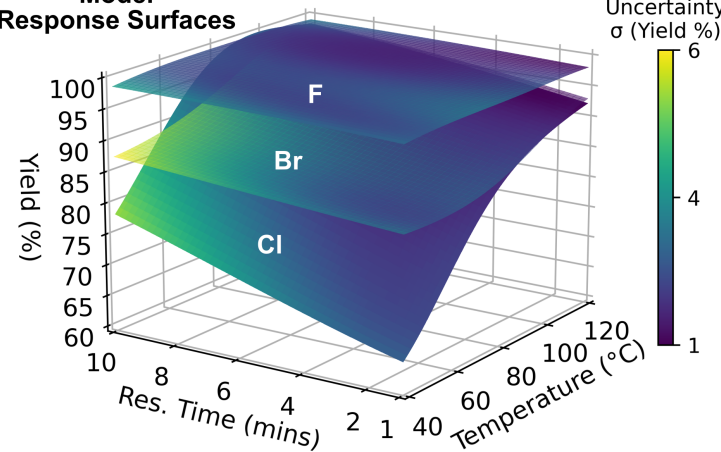

Figure 4. $S_{N} A r$ multi-objective optimization campaign. (A) Platform configuration. (B) Reaction scheme, optimization variables, and objective functions. (C) Objective values versus experiment number. (D) 3D plot of objective values. (E) 3D scatter plot showing continuous variable values explored. (F) Yield response surfaces generated using Gaussian process models fitted to experimental data. Equiv of 1 and DIPEA set to 1.1 to enable visualization.

Thirty experiments (Figure 4C-E, tabulated results in SI) were run continuously over 10 hours (3 experiments/hour) in a closed-loop manner (automated design and execution of experiments, reaction sampling, data processing and feedback). The initialization phase, which aims to conduct a preliminary 
scan of the design space, contained 9 experiments evenly divided between the three leaving groups ( 3 each). In the refinement phase, the algorithm proposed one new experiment at a time with the aim of experimentation specifically focused in regions where optimal values for all three objectives could be simultaneously achieved.

Objective values obtained over the course of the 30 experiments are plotted (Figure 4C-D) with data points color-coded by leaving group. In the initialization phase (Figure $4 \mathrm{C}$ ), yields ranged from $84 \%$ to nearly quantitative $(>98 \%)$ when $2 \mathrm{c}$ was employed as the starting material (orange points). As a result of the relatively high yields for all potential starting material candidates, the cost per mol product depended primarily on the cost differences between leaving group identity. This is visually apparent as $2 a(\sim \$ 400 / \mathrm{mol})$, $\mathbf{2 b}(\sim \$ 500 / \mathrm{mol})$, and $\mathbf{2 c}(\sim \$ 600 / \mathrm{mol})$ data points are clustered in bottom, middle, and top regions of the cost axis.

The utility of the multi-objective algorithm becomes evident in the refinement phase. With regards to the productivity objective, the initialization phase provided throughput values between $0.6-2.6 \mathrm{~g} / \mathrm{h}$ whereas the refinement phase led to a significant improvement (Figure 4C) with most experiments operating in the 5-6 $\mathrm{g} / \mathrm{h}$ range. This corresponds to more points populating the right-hand portion of the 3D scatter plot (Figure 4D) where productivity is high. Furthermore, in contrast to the initialization phase where the goal is to scan the chemical design space, the algorithm's goal in the refinement phase is to identify conditions where all three objective values are optimized simultaneously. Consequently, the majority of experiments in the refinement phase have high yields as well as high productivities.

For certain systems, multi-objective optimization can lead to situations where one objective cannot be improved without making another one worse off. The set of optimal points for which this trade-off exists is known as the Pareto front. ${ }^{16}$ Instead of fixing the weight (relative importance) of each objective for the campaign which would result in the identification of one optimal point along the Pareto front, the Dragonfly algorithm samples different weights at each refinement iteration which enables it to find multiple Pareto optimal points. ${ }^{39}$ For this $\mathrm{S}_{N} \mathrm{Ar}$ reaction, the data show that $2 \mathrm{c}$ (orange, $\mathrm{F}$ leaving group) led to the highest yield and productivity combinations (e.g., expt. 30: $98.3 \%, 5.97 \mathrm{~g} / \mathrm{h}, \$ 595 / \mathrm{mol}$ ). However, 2a (blue, Cl leaving group) conditions were $\sim 33 \%$ cheaper but came with the trade-off of slightly lower yields and productivities (e.g., expt. 27: $93.8 \%, 5.70 \mathrm{~g} / \mathrm{h}, \$ 414 / \mathrm{mol}$ ). Both these conditions are Pareto-optimal and it is up to the experimenter to decide which point is optimal for their specific context given other process considerations (e.g., purity requirements, separations).

To visualize the impact of continuous variables, the experimental data are plotted (Figure 4E) with respect to the three most influential continuous variables (temperature, time, equiv of 1) where a data point's color and size correlate with yield and productivity, respectively. Experiments with 2c (orange lines) resulted in nearly quantitative yields even at mild reaction conditions, whereas $\mathbf{2 a}$ (blue) and $\mathbf{2 b}$ (green) required higher temperatures and longer residence times to promote reactivity. Data points with the largest size (highest productivity) are concentrated on the right face of the plot corresponding to the shortest residence time of 1 minute.

The Gaussian process (GP) mathematical model that underlies the Bayesian algorithm was useful not only during the optimization, but also after the fact for process characterization. By regressing the GP model to the experimental data and evaluating it over the design space, yield response surfaces for the three leaving groups were generated (Figure 4F). The surfaces represent the predicted yield (GP mean value) and the color reflects the local model uncertainty (GP standard deviation) at that point. The flatness of the $2 \mathrm{c}$ surface reflects the robust reaction rate even at low temperature, while the curvature of the $\mathbf{2} \mathbf{a}$ and $\mathbf{2} \mathbf{b}$ surfaces illustrates the significant rate acceleration that increased temperature provided. Model uncertainty is lowest (dark blue) in the more optimal regions of the design space preferentially explored by the algorithm, and uncertainty is greatest (most yellow) at low temperature and long residence time since very few experiments were sampled from this suboptimal design region. It is worth noting that if the experimenter wanted to improve model accuracy even further for process modeling purposes, the ability to quantify local uncertainty using the GP model can be leveraged to perform post-optimization experiments in regions with the highest uncertainty. 
Multistep Downstream Process Optimization Campaign

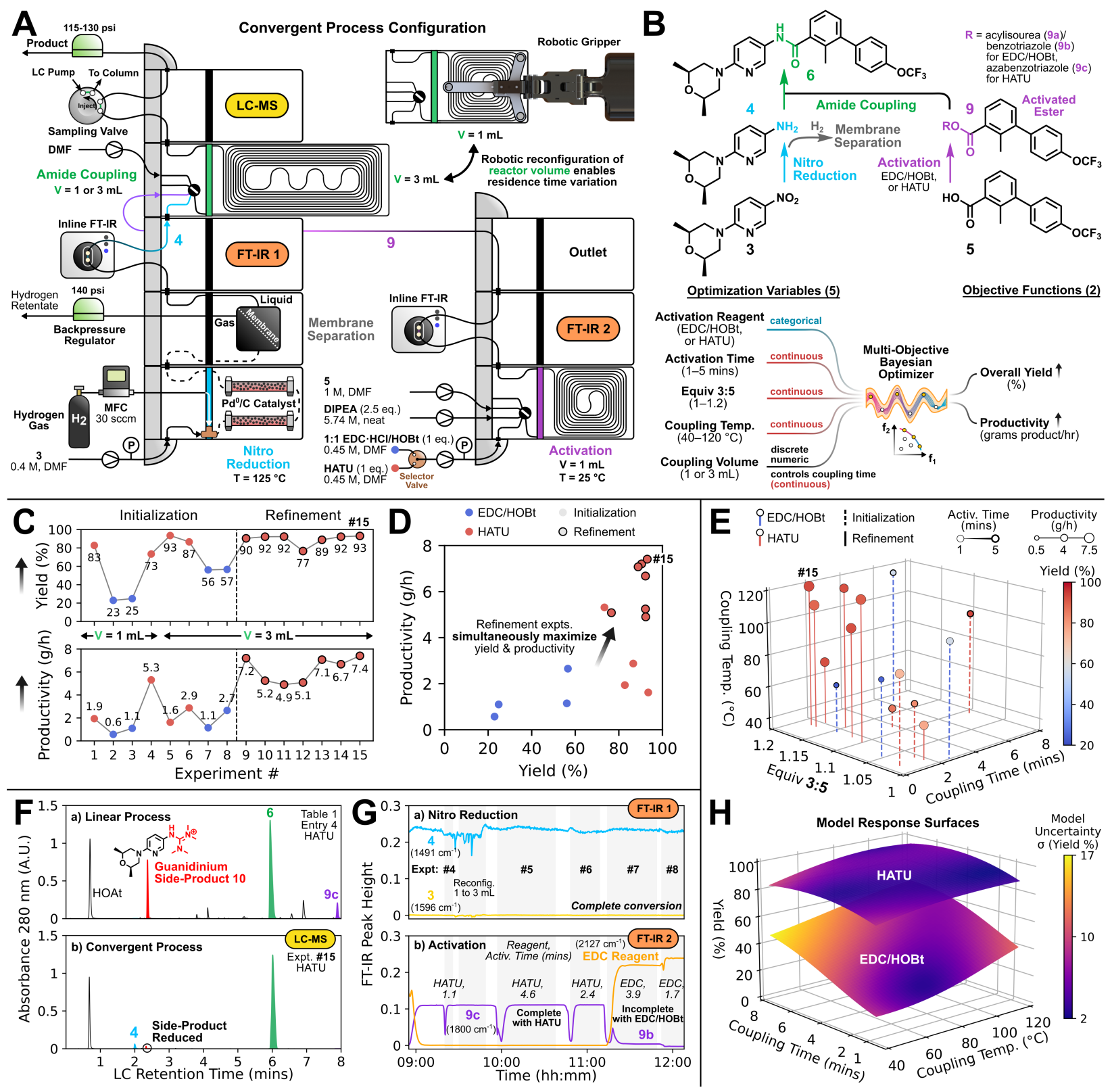

Figure 5. Multistep downstream process optimization campaign for sonidegib synthesis. (A) Platform configuration for convergent process. (B) Process scheme, optimization variables, and objective functions. (C) Objective values versus experiment number. (D) 2D plot of objective values. (E) 3D scatter plot showing continuous variable values explored. (F) LC chromatograms from (a) linear and (b) convergent process. (G) FT-IR timecourse data from (a) nitro reduction of 3 and (b) activation of 5. (H) Yield response surfaces generated using Gaussian process models fitted to experimental data. Activation time and equiv 3:5 set to $1 \mathrm{~min}$ and 1.1 equiv to enable visualization. Abbreviations: HOAt (1-hydroxy-7-azabenzotriazole).

Following the $S_{N} A r$ campaign, we optimized the multistep downstream process (Figure 5) involving nitro reduction, gas-liquid separation, and amide coupling for converting purified $S_{N} A r$ product 3 into sonidegib 6. An important change was made to the process configuration, however, based on prior results. During the fully telescoped process experiments (Figure 3, Table 1), we noticed a significant side-product in the final amide coupling LC-MS data (Figure $5 \mathrm{~F}(\mathrm{a})$ ) when HATU was employed as the coupling reagent. Based 
on our understanding of the reaction mechanism (see $\mathrm{SI}),{ }^{51}$ this side-product was assigned as guanidinium 10, formed by an undesired reaction between amine $\mathbf{4}$ and HATU which occurred due to simultaneous addition of the amide coupling streams (Figure $3 \mathrm{~A}$ ). This side-reaction can be suppressed by adding the amine after HATU is consumed in the desired activation reaction with carboxylic acid $\mathbf{5}$. Therefore, the activation and amide coupling steps were performed sequentially in separate reactors to control the order of addition. To do so, we took advantage of the synthesis platform's flexibility by switching from a linear to a convergent process configuration (Figure 5A-B) containing two parallel branches (nitro reduction to form amine 4, and activation to form the activated ester $9 \mathrm{~b}$ or 9c) that merge downstream for coupling. Two FTIR modules were deployed to monitor both the activation reaction and the nitro reduction Pd catalyst stability in real-time over the course of the campaign. A spherical $\mathrm{Pd} / \mathrm{C}$ catalyst ${ }^{48}$ (Heraeus $\mathrm{GmbH}$ ) with high surface area and low pressure drop was used for the nitro reduction, which was operated at high temperature $\left(125^{\circ} \mathrm{C}\right)$ to minimize the likelihood of deactivation. Overall yield of sonidegib 6 was determined via an LC-MS module after the amide coupling reactor.

Five optimization variables and two objective functions (Figure 5B) were considered for the multistep campaign. The activation reagent (EDC/HOBt, or HATU) and activation residence time were categorical and continuous variables, respectively. Equivalents of nitro $\mathbf{3}$ with respect to carboxylic acid $\mathbf{5}$ was included as a continuous variable since amide couplings are often performed with a slight excess of the amine. Since the flow rate of 3 was fully specified by the 3:5 stoichiometry and acid flow rate in the activation reactor, the reduction residence time also varied based on equivalents of 3 and activation time. For the final amide coupling step, in addition to including temperature as a continuous variable, reactor volume (1 or $3 \mathrm{~mL})$ was a discrete numeric variable that was automatically reconfigured by the robotic gantry arm during the campaign to provide an independent degree of freedom for accessing shorter and longer residence times without altering upstream flow rates. Overall yield and productivity of sonidegib 6 were chosen as the objective functions, while cost was not included in this case since both coupling reagents were similarly priced.

Fifteen total experiments were run over 13 hours with 8 initialization and 7 refinement experiments (Figure $5 \mathrm{C}-\mathrm{G}$, tabulated results in $\mathrm{SI}$ ). The initialization runs were evenly divided between the two activation reagents and reactor volumes (Figure $5 \mathrm{C}$ ), and higher yields were observed with HATU (red points, 7393\%) than EDC/HOBt (blue points, 23-56\%). After the first 4 experiments which utilized a $1 \mathrm{~mL}$ reactor for the amide coupling, the system performed a mid-run robotic reconfiguration from the 1 to $3 \mathrm{~mL}$ reactor (see Supplementary Movie) and brought the process back up to resume the campaign. The ability to change reactor volume helped us investigate the effect of coupling time independently of other variables. In this case, longer reaction times provided by the larger volume increased conversion and yield. This is particularly evident when comparing EDC/HOBt experiment $3(1 \mathrm{~mL}, 1 \mathrm{~min}, 25 \%$ yield, $1.1 \mathrm{~g} / \mathrm{h})$ and experiment $8(3 \mathrm{~mL}, 3 \mathrm{mins}, 57 \%$ yield, $2.65 \mathrm{~g} / \mathrm{h})$ which employed otherwise similar conditions.

For all the refinement experiments (9-15), the algorithm proposed HATU and the $3 \mathrm{~mL}$ reactor. Compared to the initialization (e.g., expt. 5, 93\% yield, $1.6 \mathrm{~g} / \mathrm{h}$ ), the multi-objective optimization algorithm identified conditions in the refinement phase with simultaneously high yields and productivities (optimal expt. 15, 93\% yield, $7.4 \mathrm{~g} / \mathrm{h}$ ) (Figure 5C-D). When plotting the data (Figure 5E) with respect to the three most impactful continuous variables (coupling temperature, coupling time, equiv 3:5), the refinement experiments (points with solid vertical lines) generally employed faster coupling times and elevated temperatures to boost the reaction rate, yield, and productivity. The $3 \mathrm{~mL}$ reactor was key to providing sufficiently long residence times $(\sim 1.6-2$ mins $)$ at high flow rates to achieve greater conversion compared to the $1 \mathrm{~mL}$ reactor. Yield response surfaces (Figure $5 \mathrm{H}$ ) generated using the Gaussian process model fitted to the experimental data help visualize the impact of coupling temperature and time. Model uncertainty is generally much lower (dark purple) for HATU than EDC/HOBt (more orange/yellow) since 11 out of 15 experiments were conducted with the optimal HATU reagent.

Representative analytical data (Figure 5F-G) collected by the integrated LC-MS and FT-IR modules provided insight into the individual reactions within the multistep process. Comparing the LC profiles from the fully telescoped linear process and convergent process (Figure $5 \mathrm{~F}$ ) showed that guanidinium impurity 10 (red peak) formed by the undesired side-reaction between HATU and amine 4 was significantly reduced in the convergent process. Monitoring the activation reactor outlet using FT-IR module 2 (Figure 5G(b)) 
revealed that the steady-state concentration of the HATU activated ester 9c (purple profile) reached the same plateau for all HATU experiments which ranged from 1 to 5 mins of activation time. This indicated that activation of acid $\mathbf{5}$ with HATU was rapid and proceeded essentially to full conversion even at 1 minute. As a result of the controlled order of addition with the convergent process, minimal HATU proceeded downstream, which suppressed guanidinium side-product 10 and increased overall process yield. The first FT-IR module, which analyzed the liquid permeate from the nitro reduction and gas-liquid separator, played a key role in helping us verify in real-time that the $\mathrm{Pd}$ catalyst activity was stable as the campaign progressed. The diagnostic IR signal from 3 (Figure 5G(a), yellow profile) was at the baseline, which indicated high levels of conversion even at the highest liquid flow rates $(\sim 0.8 \mathrm{~mL} / \mathrm{min})$. This was corroborated by the complementary LC-MS module downstream which also revealed complete reduction to amine 4. The enabling features of continuous processing for hydrogenation (enhanced gas-liquid-solid mass transfer, high local catalyst loading, and prompt telescoping of the oxidation-sensitive amine into the amide coupling) contributed to realizing high nitro reduction throughput and sonidegib productivity.

\section{Conclusion}

A CASP-proposed and human-refined multistep synthesis recipe for an exemplary small molecule, sonidegib 6, was experimentally optimized on a modular, robotic flow synthesis platform. Integrated analytics (FT-IR, LC-MS) facilitated data-rich experimentation and monitoring of multiple reactions ( $\mathrm{S}_{N} \mathrm{Ar}$, nitro reduction, amide coupling) for thorough process understanding. Automation scripts for reaction execution, sampling, and data processing helped accelerate and reduce manual effort during experimentation. By utilizing a multi-objective Bayesian optimization algorithm that iteratively proposed new conditions to evaluate in order to optimize several objectives simultaneously (yield, productivity, cost), optimal settings for categorical (reagent choice) and continuous (temperature, time, stoichiometry) reaction conditions were identified to complete the synthesis recipe as well as to generate predictive mathematical process models. The platform's hardware features, such as robotically reconfigurable reactor volumes (1 and $3 \mathrm{~mL}$ ) and convergent synthesis capability, were essential for allowing variation of downstream residence time in multistep flow synthesis and controlling the order of addition to minimize unproductive reactivity.

This work not only explored how algorithmic optimization can help fill in process details when integrating CASP tools with recipe-driven synthesis platforms, but also identified several areas where human input is still needed. In multistep syntheses, any chemical incompatibilities between reagents across steps that are hard to anticipate a priori (e.g., $\mathrm{S}_{N} A r$ byproduct leading to catalyst deactivation in the sonidegib 6 case study) pose a major challenge for one-pot or telescoped synthesis. Such cases may necessitate intermediate purification procedures which can be challenging to predict or develop, especially when nonchromatographic. Furthermore, procedural details such as concentration and solubility require additional experiments, while knowledge of reaction mechanism and human interpretation of analytical data are needed to inform order of reagent addition and strategies to eliminate side-reactions. Due to limited data availability, it is currently not possible to plan every detail required to execute a synthesis without human intervention. However, this work exemplifies how machine assistance in the repetitive aspects of initial formulation, experimental execution, and data collection can help us focus on the application of domain knowledge, critical interpretation of data, and creative problem-solving where human input still provides the greatest utility. 


\section{Supporting Information}

- Supporting Information (PDF): Additional details on automated synthesis platform, computer-aided synthesis planning recommendations, optimization algorithm, materials and methods, and experimental results

- Supplementary Movie (MP4): Movie summarizing results, including videos of robotic synthesis platform in operation during optimization campaigns

- Code availability: Python code to control common lab equipment connected to the platform (e.g., pumps, valves, HPLC) and a tutorial notebook demonstrating how to use the Bayesian optimization algorithm (including visualization of model response surfaces) are available through GitHub at https://github.com/anirudh-nambiar/make-it-system.

\section{Conflict of Interest}

The authors declare no competing financial interest.

\section{Acknowledgments}

This work was funded by the DARPA Make-It and Accelerated Molecular Discovery programs under contracts ARO W911NF-16-2-0023 and HR00111920025, respectively. We thank P. Mahesh and B. OwenBlock for their contributions to the synthesis platform, Dr. M. Fortunato for help with the ASKCOS software, Dr. A. Adamo for guidance regarding membrane separation, and Dr. R. Gérardy and S. J. Mear for help with column chromatography. We thank Heraeus Deutschland $\mathrm{GmbH} \&$ Co. KG for generously providing spherical $\mathrm{Pd} / \mathrm{C}$ catalyst samples.

\section{References}

(1) Thakkar, A.; Johansson, S.; Jorner, K.; Buttar, D.; Reymond, J.-L.; Engkvist, O. Artificial Intelligence and Automation in Computer Aided Synthesis Planning. React. Chem. Eng. 2021, 6, $27-51$.

(2) Struble, T. J.; Alvarez, J. C.; Brown, S. P.; Chytil, M.; Cisar, J.; Desjarlais, R. L.; Engkvist, O.; Frank, S. A.; Greve, D. R.; Griffin, D. J.; Hou, X.; Johannes, J. W.; Kreatsoulas, C.; Lahue, B.; Mathea, M.; Mogk, G.; Nicolaou, C. A.; Palmer, A. D.; Price, D. J.; Robinson, R. I.; Salentin, S.; Xing, L.; Jaakkola, T.; Green, W. H.; Barzilay, R.; Coley, C. W.; Jensen, K. F. Current and Future Roles of Artificial Intelligence in Medicinal Chemistry Synthesis. J. Med. Chem. 2020, 63, 8667-8682.

(3) Godfrey, A. G.; Masquelin, T.; Hemmerle, H. A Remote-Controlled Adaptive Medchem Lab: An Innovative Approach to Enable Drug Discovery in the 21st Century. Drug Discov. Today 2013, 18, 795-802.

(4) Bédard, A. C.; Adamo, A.; Aroh, K. C.; Russell, M. G.; Bedermann, A. A.; Torosian, J.; Yue, B.; Jensen, K. F.; Jamison, T. F. Reconfigurable System for Automated Optimization of Diverse Chemical Reactions. Science 2018, 361, 1220-1225.

(5) Steiner, S.; Wolf, J.; Glatzel, S.; Andreou, A.; Granda, J. M.; Keenan, G.; Hinkley, T.; AragonCamarasa, G.; Kitson, P. J.; Angelone, D.; Cronin, L. Organic Synthesis in a Modular Robotic System Driven by a Chemical Programming Language. Science 2019, 363, eaav2211.

(6) Coley, C. W.; Thomas, D. A.; Lummiss, J. A. M.; Jaworski, J. N.; Breen, C. P.; Schultz, V.; Hart, T.; Fishman, J. S.; Rogers, L.; Gao, H.; Hicklin, R. W.; Plehiers, P. P.; Byington, J.; Piotti, J. S.; Green, W. H.; John Hart, A.; Jamison, T. F.; Jensen, K. F. A Robotic Platform for Flow Synthesis of Organic Compounds Informed by Al Planning. Science 2019, 365, eaax1566.

(7) Collins, N.; Stout, D.; Lim, J.-P.; Malerich, J. P.; White, J.; Madrid, P. B.; Latendresse, M.; Krieger, D.; Szeto, J.; Vu, V.-A.; Rucker, K.; Deleo, M.; Gorfu, Y.; Krummenacker, M.; Hokama, L.; Karp, P.; Mallya, S. Fully Automated Chemical Synthesis: Toward the Universal Synthesizer. Org. Process Res. Dev. 2020, 24, 2064-2077. 
(8) Chatterjee, S.; Guidi, M.; Seeberger, P. H.; Gilmore, K. Automated Radial Synthesis of Organic Molecules. Nature 2020, 579, 379-384.

(9) Sagmeister, P.; Lebl, R.; Castillo, I.; Rehrl, J.; Kruisz, J.; Sipek, M.; Horn, M.; Sacher, S.; Cantillo, D.; Williams, J.; Kappe, C. O. Advanced Real-Time Process Analytics for Multistep Synthesis in Continuous Flow. Angew. Chem. Int. Ed. 2021, 60, 8139-8148.

(10) Liu, C.; Xie, J.; Wu, W.; Wang, M.; Chen, W.; Idres, S. B.; Rong, J.; Deng, L. W.; Khan, S. A.; Wu, J. Automated Synthesis of Prexasertib and Derivatives Enabled by Continuous-Flow Solid-Phase Synthesis. Nat. Chem. 2021, 13, 451-457.

(11) IBM RXN for Chemistry. https://rxn.res.ibm.com/rxn/robo-rxn/welcome (accessed Feb 23, 2022).

(12) Klucznik, T.; Mikulak-Klucznik, B.; McCormack, M. P.; Lima, H.; Szymkuć, S.; Bhowmick, M.; Molga, K.; Zhou, Y.; Rickershauser, L.; Gajewska, E. P.; Toutchkine, A.; Dittwald, P.; Startek, M. P.; Kirkovits, G. J.; Roszak, R.; Adamski, A.; Sieredzińska, B.; Mrksich, M.; Trice, S. L. J.; Grzybowski, B. A. Efficient Syntheses of Diverse, Medicinally Relevant Targets Planned by Computer and Executed in the Laboratory. Chem 2018, 4, 522-532.

(13) Mikulak-Klucznik, B.; Gołębiowska, P.; Bayly, A. A.; Popik, O.; Klucznik, T.; Szymkuć, S.; Gajewska, E. P.; Dittwald, P.; Staszewska-Krajewska, O.; Beker, W.; Badowski, T.; Scheidt, K. A.; Molga, K.; Mlynarski, J.; Mrksich, M.; Grzybowski, B. A. Computational Planning of the Synthesis of Complex Natural Products. Nature 2020, 588, 83-88.

(14) Lin, Y.; Zhang, Z.; Mahjour, B.; Wang, D.; Zhang, R.; Shim, E.; McGrath, A.; Shen, Y.; Brugger, N.; Turnbull, R.; Trice, S.; Jasty, S.; Cernak, T. Reinforcing the Supply Chain of Umifenovir and Other Antiviral Drugs with Retrosynthetic Software. Nat. Commun. 2021, 12, 1-8.

(15) Coley, C. W.; Eyke, N. S.; Jensen, K. F. Autonomous Discovery in the Chemical Sciences Part II: Outlook. Angew. Chemie Int. Ed. 2020, 59, 23414-23436.

(16) Clayton, A. D.; Manson, J. A.; Taylor, C. J.; Chamberlain, T. W.; Taylor, B. A.; Clemens, G.; Bourne, R. A. Algorithms for the Self-Optimisation of Chemical Reactions. React. Chem. Eng. 2019, 4, 15451554.

(17) Mateos, C.; Nieves-Remacha, M. J.; Rincón, J. A. Automated Platforms for Reaction SelfOptimization in Flow. React. Chem. Eng. 2019, 4, 1536-1544.

(18) Reizman, B. J.; Wang, Y. M.; Buchwald, S. L.; Jensen, K. F. Suzuki-Miyaura Cross-Coupling Optimization Enabled by Automated Feedback. React. Chem. Eng. 2016, 1, 658-666.

(19) Schweidtmann, A. M.; Clayton, A. D.; Holmes, N.; Bradford, E.; Bourne, R. A.; Lapkin, A. A. Machine Learning Meets Continuous Flow Chemistry: Automated Optimization towards the Pareto Front of Multiple Objectives. Chem. Eng. J. 2018, 352, 277-282.

(20) Cortés-Borda, D.; Wimmer, E.; Gouilleux, B.; Barré, E.; Oger, N.; Goulamaly, L.; Peault, L.; Charrier, B.; Truchet, C.; Giraudeau, P.; Rodriguez-Zubiri, M.; Le Grognec, E.; Felpin, F.-X. An Autonomous Self-Optimizing Flow Reactor for the Synthesis of Natural Product Carpanone. J. Org. Chem. 2018, 83, 14286-14299.

(21) Shields, B. J.; Stevens, J.; Li, J.; Parasram, M.; Damani, F.; Alvarado, J. I. M.; Janey, J. M.; Adams, R. P.; Doyle, A. G. Bayesian Reaction Optimization as a Tool for Chemical Synthesis. Nature 2021, 590, 89-96.

(22) Christensen, M.; Yunker, L. P. E.; Adedeji, F.; Häse, F.; Roch, L. M.; Gensch, T.; dos Passos Gomes, G.; Zepel, T.; Sigman, M. S.; Aspuru-Guzik, A.; Hein, J. E. Data-Science Driven Autonomous Process Optimization. Commun. Chem. 2021, 4, 1-12.

(23) Fath, V.; Kockmann, N.; Otto, J.; Röder, T. Self-Optimising Processes and Real-Time-Optimisation of Organic Syntheses in a Microreactor System Using Nelder-Mead and Design of Experiments. React. Chem. Eng. 2020, 5, 1281-1299.

(24) Reizman, B. J.; Jensen, K. F. Feedback in Flow for Accelerated Reaction Development. Acc. Chem. Res. 2016, 49, 1786-1796.

(25) Häse, F.; Roch, L. M.; Aspuru-Guzik, A. Next-Generation Experimentation with Self-Driving Laboratories. Trends Chem. 2019, 1, 282-291.

(26) Breen, C. P.; Nambiar, A. M. K.; Jamison, T. F.; Jensen, K. F. Ready, Set, Flow! Automated Continuous Synthesis and Optimization. Trends Chem. 2021, 3, 373-386.

(27) Clayton, A. D.; Power, L. A.; Reynolds, W. R.; Ainsworth, C.; Hose, D. R. J.; Jones, M. F.; Chamberlain, T. W.; Blacker, A. J.; Bourne, R. A. Self-Optimising Reactive Extractions: Towards the Efficient Development of Multi-Step Continuous Flow Processes. J. Flow Chem. 2020, 10, 199-206. 
(28) Clayton, A. D.; Schweidtmann, A. M.; Clemens, G.; Manson, J. A.; Taylor, C. J.; Niño, C. G.; Chamberlain, T. W.; Kapur, N.; Blacker, A. J.; Lapkin, A. A.; Bourne, R. A. Automated SelfOptimisation of Multi-Step Reaction and Separation Processes Using Machine Learning. Chem. Eng. J. 2020, 384, 123340.

(29) ASKCOS Homepage. https://askcos.mit.edu/ (accessed Feb 23, 2022).

(30) Pan, S.; Wu, X.; Jiang, J.; Gao, W.; Wan, Y.; Cheng, D.; Han, D.; Liu, J.; Englund, N. P.; Wang, Y.; Peukert, S.; Miller-Moslin, K.; Yuan, J.; Guo, R.; Matsumoto, M.; Vattay, A.; Jiang, Y.; Tsao, J.; Sun, F.; Pferdekamper, A. C.; Dodd, S.; Tuntland, T.; Maniara, W.; Kelleher, J. F.; Yao, Y. M.; Warmuth, M.; Williams, J.; Dorsch, M. Discovery of NVP-LDE225, a Potent and Selective Smoothened Antagonist. ACS Med. Chem. Lett. 2010, 1, 130-134.

(31) Jain, S.; Song, R.; Xie, J. Sonidegib: Mechanism of Action, Pharmacology, and Clinical Utility for Advanced Basal Cell Carcinomas. Onco Targets Ther. 2017, 10, 1645-1653.

(32) Gutmann, B.; Cantillo, D.; Kappe, C. O. Continuous-Flow Technology - A Tool for the Safe Manufacturing of Active Pharmaceutical Ingredients. Angew. Chemie Int. Ed. 2015, 54, 6688-6728.

(33) Plutschack, M. B.; Pieber, B.; Gilmore, K.; Seeberger, P. H. The Hitchhiker's Guide to Flow Chemistry. Chem. Rev. 2017, 117, 11796-11893.

(34) Jensen, K. F. Flow Chemistry-Microreaction Technology Comes of Age. AlChE J. 2017, 63, 858869.

(35) Britton, J.; Raston, C. L. Multi-Step Continuous-Flow Synthesis. Chem. Soc. Rev. 2017, 46, 12501271.

(36) Papoulias, S. A.; Grossmann, I. E. A Structural Optimization Approach in Process Synthesis-III. Total Processing Systems. Comput. Chem. Eng. 1983, 7, 723-734.

(37) Dietz, T.; Klamroth, K.; Kraus, K.; Ruzika, S.; Schäfer, L. E.; Schulze, B.; Stiglmayr, M.; Wiecek, M. M. Introducing Multiobjective Complex Systems. Eur. J. Oper. Res. 2020, 280, 581-596.

(38) Kandasamy, K.; Vysyaraju, K. R.; Neiswanger, W.; Paria, B.; Collins, C. R.; Schneider, J.; Póczos, B.; Xing, E. P. Tuning Hyperparameters without Grad Students: Scalable and Robust Bayesian Optimisation with Dragonfly. J. Mach. Learn. Res. 2020, 21, 1-25.

(39) Paria, B.; Kandasamy, K.; Póczos, B. A Flexible Framework for Multi-Objective Bayesian Optimization Using Random Scalarizations. arXiv 2018. https://arxiv.org/abs/1805.12168 (accessed Feb 23, 2022).

(40) https://github.com/dragonfly/dragonfly (accessed Feb 23, 2022).

(41) Frazier, P. I. A Tutorial on Bayesian Optimization. arXiv 2018. https://arxiv.org/abs/1807.02811 (accessed Feb 23, 2022).

(42) Irfan, M.; Glasnov, T. N.; Kappe, C. O. Heterogeneous Catalytic Hydrogenation Reactions in Continuous-Flow Reactors. ChemSusChem 2011, 4, 300-316.

(43) Pagliaro, M.; Pandarus, V.; Béland, F.; Ciriminna, R.; Palmisano, G.; Carà, P. D. A New Class of Heterogeneous Pd Catalysts for Synthetic Organic Chemistry. Catal. Sci. Technol. 2011, 1, 736-739.

(44) Yang, C.; Teixeira, A. R.; Shi, Y.; Born, S. C.; Lin, H.; Li Song, Y.; Martin, B.; Schenkel, B.; Peer Lachegurabi, M.; Jensen, K. F. Catalytic Hydrogenation of: N -4-Nitrophenyl Nicotinamide in a MicroPacked Bed Reactor. Green Chem. 2018, 20, 886-893.

(45) Hornung, C. H.; Nguyen, X.; Carafa, A.; Gardiner, J.; Urban, A.; Fraser, D.; Horne, M. D.; Gunasegaram, D. R.; Tsanaktsidis, J. Use of Catalytic Static Mixers for Continuous Flow Gas-Liquid and Transfer Hydrogenations in Organic Synthesis. Org. Process Res. Dev. 2017, 21, 1311-1319.

(46) Gardiner, J.; Nguyen, X.; Genet, C.; Horne, M. D.; Hornung, C. H.; Tsanaktsidis, J. Catalytic Static Mixers for the Continuous Flow Hydrogenation of a Key Intermediate of Linezolid (Zyvox). Org. Process Res. Dev. 2018, 22, 1448-1452.

(47) Lebl, R.; Bachmann, S.; Tosatti, P.; Sedelmeier, J.; Püntener, K.; Williams, J. D.; Kappe, C. O. Catalytic Static Mixer-Enabled Hydrogenation of a Key Fenebrutinib Intermediate: Real-Time Analysis for a Stable and Scalable Process. Org. Process Res. Dev. 2021, 25, 1988-1995.

(48) Fernandez-Puertas, E.; Robinson, A. J.; Robinson, H.; Sathiyalingam, S.; Stubbs, H.; Edwards, L. J. Evaluation and Screening of Spherical $\mathrm{Pd} / \mathrm{C}$ for Use as a Catalyst in Pharmaceutical-Scale Continuous Hydrogenations. Org. Process Res. Dev. 2020, 24, 2147-2156.

(49) van Gelder, K. B.; Damhof, J. K.; Kroijenga, P. J.; Westerterp, K. R. Three-Phase Packed Bed Reactor with an Evaporating Solvent-I. Experimental: The Hydrogenation of 2,4,6-Trinitrotoluene in Methanol. Chem. Eng. Sci. 1990, 45, 3159-3170. 
(50) Figueras, F.; Coq, B. Hydrogenation and Hydrogenolysis of Nitro-, Nitroso-, Azo-, Azoxy- and Other Nitrogen-Containing Compounds on Palladium. J. Mol. Catal. A Chem. 2001, 173, 223-230.

(51) Valeur, E.; Bradley, M. Amide Bond Formation: Beyond the Myth of Coupling Reagents. Chem. Soc. Rev. 2009, 38, 606-631. 